\title{
Mental health in the UK during the COVID-19 pandemic: early observations
}

\section{Brief title: Mental Health in the UK and COVID-19}

${ }^{1}$ Ru Jia MSc, ${ }^{1}$ Kieran Ayling PhD, ${ }^{2}$ Trudie Chalder PhD, ${ }^{1}$ Adam Massey PhD, ${ }^{3}$ Elizabeth Broadbent PhD, ${ }^{1}$ Carol Coupland PhD, ${ }^{1 *}$ Kavita Vedhara PhD

\author{
${ }^{1}$ Division of Primary Care \\ University of Nottingham \\ University Park \\ Nottingham, NG7 2RD
}

${ }^{2}$ Department of Psychological Medicine, Institute of Psychiatry, Psychology \& Neuroscience,

King's College London,

16, De Crespigny Park,

London, SE5 8AF,

United Kingdom.

${ }^{3}$ Department of Psychological Medicine,

University of Auckland,

Private bag 92019, Auckland,

New Zealand.

* Author to whom all correspondence should be addressed: Professor Kavita Vedhara, Division of Primary Care, University of Nottingham, University Park, Nottingham, NG7 2RD, UK.

Kavita.vedhara@nottingham.ac.uk; Tel +44 1158466931 


\begin{abstract}

\section{Background:}

Previous pandemics have resulted in significant consequences for mental health. Here we report the mental health sequela of the COVID-19 pandemic on the UK population and examine modifiable and non-modifiable explanatory factors associated with mental health outcomes. We focus on the short-term consequences for mental health, as reported during the first four-six weeks of social distancing measures being introduced.
\end{abstract}

\title{
Methods:
}

A community cohort study was conducted with adults aged $\geq 18$ years recruited through a mainstream and social media campaign between 3/4/20-30/4/20. Consenting participants completed an online survey measuring depression, anxiety and stress and explanatory variables hypothesised to be related to these mental health outcomes.

\section{Outcomes:}

$\mathrm{N}=3097$ eligible individuals participated. The cohort was predominantly female ( $85 \%)$; mean age forty-four years; $10 \%$ from minority ethnic groups; $50 \%$ described themselves as key-workers and $20 \%$ identified as having clinical risk factors putting them at increased risk of COVID-19. Mean scores for depression, stress and anxiety significantly exceeded population norms. Analysis of non-modifiable factors indicated that being younger and female were associated with all outcomes, with the final multivariable models accounting for 7$13 \%$ of variance. When adding modifiable factors, significant independent effects emerged for positive mood, perceived loneliness and worry about getting COVID-19 for all outcomes, with the final multivariable models accounting for $54-57 \%$ of variance.

\section{Interpretation:}

Increased psychological morbidity was evident in this UK cohort, with younger people and women at particular risk. Interventions targeting perceptions of: loneliness, risk of COVID-19, worry about COVID-19, and positive mood may be effective.

\section{Acknowledgements:}

KA was supported by funding from the National Institute for Health Research School for Primary Care Research (NIHR SPCR). The views expressed are those of the author(s) and not necessarily those of the NIHR, the NHS or the Department of Health.

TC acknowledges the financial support of the Department of Health via the National Institute for Health Research (NIHR) Specialist Biomedical Research Centre for Mental Health award to the South London and Maudsley NHS Foundation Trust (SLaM) and the Institute of Psychiatry at King's College London. The views expressed are those of the authors and not necessarily those of the NHS, the NIHR or the Department of Health and Social Care. 


\section{Background}

The COVID-19 (Coronavirus, 2019) pandemic has resulted in unprecedented disruption to the fabric of society, our health service and economy. However, the multitude of challenges presented by the pandemic may also pose a significant threat to our psychological health. ${ }^{1}$ Individuals are facing a panoply of stressors including: serious illness, bereavement, social distancing, and unemployment. The consequences of these stressors for mental health will not be uniform, rather they will be influenced by a range of modifiable and non-modifiable factors. Understanding these factors will be critical in determining who is at greatest risk of mental health difficulties and potential approaches to intervention. We report here cross-sectional findings from a community cohort study designed to capture both the mental health sequela of the COVID-19 pandemic, as well as the modifiable and non-modifiable explanatory factors associated with adverse mental health outcomes. Our focus is on the immediate consequences for mental health, as reported during the first 4-6 weeks of social distancing measures being introduced in the UK.

In keeping with its recent emergence, much remains unknown about COVID-19 and its consequences. However, the expectation is that the consequences for mental health will be profound and far reaching. ${ }^{1}$ Evidence on the impact of the pandemic on people living in China attests to this possibility. For example, one study reported the prevalence of depression and anxiety in medical staff to be over 50\% and $44 \%$ respectively. ${ }^{2}$ Similarly, a study with a general population sample during Level 1 of the epidemic (classified as a 'significant' emergency) revealed that greater than $70 \%$ of participants reported moderate or high psychological symptoms of obsessive compulsion, interpersonal sensitivity, phobic anxiety, and psychoticism. ${ }^{3}$

The experience of previous pandemics also suggests that significant mental health consequences are probable. During the Middle East Respiratory Syndrome outbreak, for example, untoward psychological effects occurred rapidly: with symptoms of anxiety and anger evident after brief isolation periods of 2 weeks. ${ }^{4}$ Similarly, the Severe Acute Respiratory Syndrome epidemic was associated with a 31\% increase in suicide amongst the over 65 s in Hong Kong. ${ }^{5}$

Preliminary evidence from the UK suggests that these experiences may be replicated here. For example, two online surveys conducted in the first week after social distancing measures were introduced, highlighted some early concerns. ${ }^{1}$ One survey was conducted with people with lived experience of mental health problems and stakeholders $(\mathrm{n}=2198)$, and the other a nationally representative sample of the general population $(\mathrm{n}=1099)$. Both cohorts were asked about their concerns regarding the mental health impact of the COVID-19 pandemic. Although no validated assessments of mental health were undertaken, a common theme for both cohorts was concern about increasing levels of anxiety, and the role of social distancing measures and well-being of family members in contributing to such anxiety. Similarly, a qualitative study examining the mental health impact of social distancing measures suggested that deleterious effects were evident within 5-12 days of such measures being in place. ${ }^{6}$

So it would seem likely that we should brace ourselves for a significant increase in mental health difficulties associated with the pandemic. But who might be at greatest risk? Individuals at increased risk of the disease and/or adverse outcomes might be expected to experience greater mental health difficulties. Several such groups have now been identified. For example, the death rate is known to be higher in men and older individuals. ${ }^{7,8}$ The latter also being more likely to have co-existing conditions and be socially-isolated through shielding. The ethnic diversity of countries such as the US and UK has also highlighted that individuals from Black, Asian and Minority Ethnic (BAME) backgrounds appear to be affected disproportionately by the disease. ${ }^{9}$ Finally, recent UK data suggest that some key-workers, in particular those in social care, are at greater risk of COVID-19 related mortality. ${ }^{7}$ This combined with evidence from China of increased anxiety and depression among health care workers, ${ }^{3}$ suggests that key-workers may also be at greater risk of adverse mental health outcomes.

The aforementioned factors are, however, largely non-modifiable. Do modifiable risk factors exist which could be targets for intervention? Stress and coping theory. ${ }^{10}$ attests that emotional responses to challenging situations vary according to both our appraisal of stressors and the availability of psychological and social resources. Cognitions are central to our appraisal process and, in the current context, one might expect that individuals who appraise that they are at greatest risk of disease may have poorer mental health outcomes. Evidence from the Netherlands during the H1N1 pandemic supports this hypothesis, with prospective data indicating that levels of anxiety declined as perceptions of the risk of acquiring severe disease declined. ${ }^{11}$ Another relevant feature of our appraisal process concerns the extent to which we attend to and worry about threats to our health. This is 
particularly pertinent in contexts where individuals receive health threat information from the media, as in the current pandemic. ${ }^{12}$ Early evidence of this mechanism has emerged in the COVID-19 pandemic: with frequency of exposure to information about COVID-19 on social media found to be positively associated with mental health outcomes. ${ }^{13}$

In terms of resources, social support, and its corollary loneliness, are among the best established determinants of our emotional responses to stressors. Successive systematic reviews demonstrate not only poorer mental health outcomes, but also increased morbidity and mortality in individuals who perceive themselves to be more lonely and lacking in support. ${ }^{14,15}$ A determinant also attracting attention is positive mood. It is now recognised that positive mood is not just the opposite of negative mood, but may in fact confer direct effects on well-being as well as protective effects in challenging situations. ${ }^{10,16}$ In terms of mental health outcomes, evidence suggests that the existence of positive mood reduces the risk of mood disorders by $28 \%$ and anxiety disorders by $53 \%$, and also influences recovery from some mental health conditions. ${ }^{17,18}$

Taken together there is an urgent need to report evidence on the prevalence of mental health problems during the COVID-19 pandemic, to understand who may be at greatest risk, and to explore the psychological and social resources that may mitigate this risk. To that end, we report cross sectional findings from a community cohort survey conducted between $3^{\text {rd }}$ and $30^{\text {th }}$ April 2020 which coincided with the first 4-6 weeks of social distancing measures being introduced in the UK.

\section{Methods}

\section{Recruitment and Eligibility}

Ethical approval was granted from the University of Nottingham Faculty of Medicine and Health Sciences (ref: 506-2003) and the NHS Health Research Authority (ref: 20/HRA/1858). The study was launched on 3/4/20 with participants recruited in the community through a social and mainstream media campaign involving, but not limited to, Facebook and Twitter. In addition, HRA regulatory approval enabled us to approach NHS organisations and request they advertise the research through their routine communications. Recruitment continued until 30/4/20. All media directed potential participants to the study website (www.covidstressstudy.co.uk) through which they accessed the information sheet, consent form and online survey.

Eligibility criteria specified that participants should be: aged 18 and over; able to give informed consent; able to read English; residing in the UK at the time of completing the survey and able to provide a sample of hair at least $1 \mathrm{~cm}$ long. The latter was collected for the determination of the stress biomarker cortisol which will be the subject of future manuscripts.

\section{Procedures}

Consenting participants completed an online survey implemented through JISC Online Survey (https://www.onlinesurveys.ac.uk/). This included validated measures capturing the mental health outcomes: anxiety $(\alpha=0 \cdot 88)$, depression $(\alpha=0 \cdot 92)$ and stress $(\alpha=0 \cdot 76) .{ }^{19-22}$ We also measured modifiable and nonmodifiable variables we hypothesised would be related to these mental health outcomes due to being (i) associated with an increased risk of contracting COVID-19 and/or adverse disease outcomes; or (ii) known to be directly associated with adverse mental health outcomes. These were: age, gender, ethnicity, key-worker status, living alone, positive mood, worry about contracting COVID-19 and perceived loneliness and risk of COVID19 (see supplementary appendix).

\section{Statistical analysis}

We first summarised the outcome variables (depression, anxiety and stress) and participant characteristics with appropriate summary statistics and examined histograms and scatterplots. To explore the associations between 
non-modifiable and modifiable explanatory factors on outcome variables we conducted univariable linear regression analyses (see supplementary appendix). Multivariable linear regression analyses were then used to explore the independent relationships of non-modifiable factors (age, gender, ethnicity, keyworker status, living alone) on outcome variables. Then, in subsequent models, modifiable explanatory factors (perceived loneliness, perceived risk of COVID-19, positive mood, worry about contracting COVID-19) were added to examine the additional and independent contribution of these factors to explaining variation in the outcome variables. The variable assessing COVID-19 worry was treated as a categorical variable in all models, with "occasional worry" treated as the reference value as this was the most common response. Assumptions of linear regression (normality and homoscedasticity of residuals, linearity with continuous variables) and presence of outliers were assessed graphically. Square root transformations were used for depression and anxiety scores to satisfy assumptions. Robustness of the models were examined by removing data points with large residuals (<-3 or $>3)$ and comparing results to the original models. In the vast majority of models, this had no substantive effect on interpretation. Thus these results are only mentioned where interpretation may be affected. Additionally, as perceived risk of getting COVID-19 was not assessed in those who thought they had had it $(n=519)$ these participants are not represented in final multivariable models. As a sensitivity analysis, models were additionally re-specified excluding this explanatory variable (see supplementary appendix).

For depression and anxiety we carried out additional analyses dichotomising according to established cut-offs (scores of 10 or greater indicating moderate or severe levels). We used multiple logistic regression to estimate odds ratios with $95 \%$ confidence intervals for their associations with non-modifiable and modifiable variables.

Statistical analyses were performed using STATA (version 16).

\section{Role of funding source}

The study sponsor did not play a role in the study design, collection; analysis, and interpretation of data; in the writing of the report; and in the decision to submit the paper for publication.

\section{Results}

\section{Cohort characteristics}

The final number of participants recruited was $n=3102$. Of these, five were ineligible due to being less than 18 years old. Thus, yielding $n=3097$ eligible participants. The largest proportion of visitors to the website came direct to the URL $(62 \% / n=15,218)$, followed by $25 \%(n=6068)$ via Facebook (the remainder through other websites). The vast majority of respondents accessed the website via a mobile phone $(70 \% / \mathrm{n}=17045)$. The survey was completed in full by $100 \%$ of those who started it, consequently there were no missing data, with the exception of age, for which 2 participants entered non-numeric values.

Table 1 summarises the main characteristics of the participants and reveals that our sample was predominantly female; with a mean age of 44 years (standard deviation=15); with participation across the UK (albeit primarily from England) and 10\%/n=296 from minority ethnic backgrounds. Fifty percent $(n=1559)$ described themselves as key-workers $(39 \% / n=1198$ identifying as working in health and social care). Twenty percent $(n=649)$ identified themselves as having clinical risk factors which would put them at increased or greatest risk of COVID-19.

Table 1: Participant Demographics $(n=3097)$

\begin{tabular}{ll}
\hline & Participants \\
\cline { 2 - 2 } & $\mathrm{n}(\%)$ \\
\hline Gender
\end{tabular}


medRxiv preprint doi: https://doi.org/10.1101/2020.05.14.20102012; this version posted May 19, 2020. The copyright holder for this preprint (which was not certified by peer review) is the author/funder, who has granted medRxiv a license to display the preprint in perpetuity.

It is made available under a CC-BY 4.0 International license .

Male

Female

Prefer not to say

Age groups (years)

$18-24$

25-34

$35-44$

$45-54$

$55-64$

65-74

$\geq 75$

Ethnicity

White - British, Irish, other

Asian/Asian British - Indian, Pakistani, Bangladeshi, other

Black/Black British - Caribbean, African, other

Chinese/Chinese British

Mixed race - White and Black/Black British

Middle Eastern/Middle Eastern British - Arab, Turkish, other

Mixed race - other

Other ethnic group

Prefer not to say

\section{Relationship status}

Single, never married

Single, divorced or widowed

In a relationship/married but living apart

In a relationship/married and cohabiting

Prefer not to say

Education (highest level of attainment)

No qualifications

Completed GSCE/CSE/O-levels or equivalent

Completed post-16 vocational course

A-levels or equivalent (at school until aged 18)

Undergraduate degree or professional qualification

Postgraduate degree

Prefer not to say

Place of residence

South West England

East Midlands

Yorkshire and Humber

North East

East of England

North West

South East England

Greater London
$476(15 \cdot 4 \%)$

$2618(84.5 \%)$

$3(0 \cdot 1 \%)$

$363(11 \cdot 7 \%)$

$528(17 \cdot 1 \%)$

635 (20.5\%)

$687(22 \cdot 2 \%)$

568 (18.3\%)

$254(8.2 \%)$

$62(2.0 \%)$

$2796(90.3 \%)$

$119(3.8 \%)$

$42(1.4 \%)$

$28(0.9 \%)$

19 (0.6\%)

$23(0.7 \%)$

$40(1.3 \%)$

$25(0.8 \%)$

$5(0 \cdot 2 \%)$

574 (18.5\%)

$263(8.5 \%)$

$254(8.2 \%)$

1981 (64.0\%)

$25(0 \cdot 8 \%)$

$33(1 \cdot 1 \%)$

$252(8.1 \%)$

$101(3.3 \%)$

$403(13.0 \%)$

$1306(42 \cdot 2 \%)$

$976(31.5 \%)$

$26(0 \cdot 8 \%)$

$241(7.8 \%)$

$762(24.6 \%)$

$293(9.5 \%)$

147 (4.8\%)

$153(4.9 \%)$

357 (11.5\%)

415 (13.4\%)

$329(10 \cdot 6 \%)$ 
West Midlands

Northern Ireland

Wales

Scotland

Key-worker status

Health, social care or relevant related support worker

Teacher or childcare worker still travelling in to work

Transport worker still travelling in to work

Food chain worker (e.g. production, sale, delivery)

Key public services worker (e.g. justice staff, religious staff, public service journalist or mortuary worker)

Local or national government worker delivering essential public services

Utility worker (e.g. energy, sewerage, postal service)

Public safety or national security worker

Worker involved in medicines or protective equipment production or distribution

Other key worker role not listed

Not a key worker

Living alone (or with others)

Living alone

Living with others

COVID-19 risk status

Most at risk (e.g. suffering from advanced cancer, severe asthma/COPD, etc.)

At increased risk (e.g., being pregnant, aged over 70, etc.)

Not at-risk
$165(5 \cdot 3 \%)$

$8(0 \cdot 3 \%)$

$73(2.4 \%)$

$154(5 \cdot 0 \%)$

$1198(38 \cdot 7 \%)$

$70(2.3 \%)$

$1(0.03 \%)$

$33(1 \cdot 1 \%)$

$22(0.7 \%)$

$41(1 \cdot 3 \%)$

$5(0 \cdot 2 \%)$

$11(0.4 \%)$

$10(0 \cdot 3 \%)$

$168(5 \cdot 4 \%)$

$1538(49 \cdot 7 \%)$

$406(13 \cdot 1 \%)$

$2691(86 \cdot 9 \%)$

$121(3.9 \%)$

$528(17 \cdot 1 \%)$

$2448(79.0 \%)$

\section{Mental health outcomes}

Table 2 summarises findings in relation to levels of stress, anxiety and depression in the cohort. The mean values for all measures indicate levels that are higher in women than men and decrease with age. Overall mean values are significantly higher than previously reported population norms ${ }^{23-25}$. For both anxiety and depression the means for the cohort were higher for both genders compared with their respective population norms, and also for all age ranges between 25-64 years. In contrast, both men and women aged over 65 years had anxiety and depression scores consistent with previous population norms. The data suggested no significant differences in stress scores by gender, despite the combined mean score exceeding the population norm.

Table 3 shows the categorisation of participants in line with established cut-offs for anxiety and depression. This shows $64 \%$ of participants reported symptoms of depression and $57 \%$ reported symptoms of anxiety. When considering the thresholds at which someone would qualify for high intensity psychological support (score of 10 or greater) in the $\mathrm{NHS},{ }^{26}$ we observe that $31.6 \%$ reported moderate to severe depression and $26 \%$ moderate to severe anxiety. 
Table 2: Depression (PHQ-9), anxiety (GAD-7) and stress (PSS-4) scores and published population normative data ${ }^{\dagger}$

\begin{tabular}{|c|c|c|c|c|c|c|c|c|c|}
\hline & \multicolumn{3}{|c|}{ PHQ-9 score } & \multicolumn{3}{|c|}{ GAD-7 score } & \multicolumn{3}{|c|}{ PSS-4 score } \\
\hline & Participants & Norms & & Participants & Norms & & Participants & Norms & \\
\hline & $\begin{array}{c}\text { Mean } \\
\text { (SD) }\end{array}$ & $\begin{array}{c}\text { Mean } \\
\text { (SD) }\end{array}$ & $\mathrm{t}$ & $\begin{array}{c}\text { Mean } \\
\text { (SD) }\end{array}$ & $\begin{array}{c}\text { Mean } \\
\text { (SD) }\end{array}$ & $\mathrm{t}$ & $\begin{array}{c}\text { Mean } \\
\text { (SD) }\end{array}$ & $\begin{array}{c}\text { Mean } \\
\text { (SD) }\end{array}$ & $\mathrm{t}$ \\
\hline Total Score & $7 \cdot 69(6 \cdot 0)$ & $2 \cdot 91(3 \cdot 5)$ & $45 \cdot 31 * * * *$ & $6 \cdot 59(5 \cdot 6)$ & $2.95(3.4)$ & $36 \cdot 52 * * * *$ & $6 \cdot 48(3 \cdot 3)$ & $6 \cdot 11(3 \cdot 1)$ & $3 \cdot 80 * * * *$ \\
\hline \multicolumn{10}{|l|}{ Gender } \\
\hline Male & $6 \cdot 49(6 \cdot 1)$ & $2 \cdot 7(3.5)$ & $18 \cdot 56 * * * *$ & $5 \cdot 22(5 \cdot 4)$ & $2 \cdot 66(3 \cdot 2)$ & $13 \cdot 77 * * * *$ & $5 \cdot 88(3 \cdot 3)$ & $5 \cdot 56(3 \cdot 0)$ & $1.57(p=0.12)$ \\
\hline Female & $7 \cdot 91(6 \cdot 0)$ & $3 \cdot 1(3 \cdot 5)$ & $35 \cdot 80 * * * *$ & $6 \cdot 84(5 \cdot 5)$ & $3 \cdot 20(3 \cdot 5)$ & $28 \cdot 83 * * * *$ & $6 \cdot 59(3 \cdot 3)$ & $6 \cdot 38(3 \cdot 2)$ & $1.73(p=0.084)$ \\
\hline \multicolumn{10}{|l|}{$\begin{array}{l}\text { Age groups } \\
\text { (years) }\end{array}$} \\
\hline $18-24$ & $11 \cdot 26(6 \cdot 4)$ &.$\cdot$ &.. & $9.04(5.9)$ &.$\cdot$ &.. & $7 \cdot 64(3 \cdot 3)$ & .. & .. \\
\hline $25-34$ & $8.74(5.9)$ & $2 \cdot 3(3 \cdot 2)$ & $22 \cdot 46 * * * *$ & $7 \cdot 73(5 \cdot 6)$ & $2 \cdot 81(3 \cdot 3)$ & $13 \cdot 85^{* * * * *}$ & $6.97(3.3)$ & .. & .. \\
\hline $35-44$ & $8 \cdot 24(6 \cdot 0)$ & $2 \cdot 6(3 \cdot 5)$ & $23.48 * * * *$ & $7 \cdot 25(5 \cdot 7)$ & $2 \cdot 82(3 \cdot 3)$ & $14.09 * * * *$ & $6 \cdot 40(3 \cdot 1)$ & .. &.. \\
\hline $45-54$ & $7 \cdot 34(5 \cdot 7)$ & $2 \cdot 8(3 \cdot 5)$ & $19 \cdot 31 * * * *$ & $6 \cdot 28(5 \cdot 3)$ & $3 \cdot 14(3 \cdot 4)$ & $10 \cdot 71 * * * *$ & $6 \cdot 06(3 \cdot 1)$ &.. &.. \\
\hline $55-64$ & $6 \cdot 32(5 \cdot 6)$ & $3 \cdot 2(3 \cdot 5)$ & $12 \cdot 90 * * * *$ & $5 \cdot 43(5 \cdot 1)$ & $3 \cdot 25(3 \cdot 6)$ & $7 \cdot 36 * * * *$ & $5 \cdot 40(3 \cdot 1)$ &.. &.. \\
\hline $65-74$ & $3 \cdot 81(4 \cdot 2)$ & $3 \cdot 3(3 \cdot 6)$ & $1 \cdot 88(p=0 \cdot 060)$ & $3 \cdot 33(3 \cdot 8)$ & $2 \cdot 79(3 \cdot 2)$ & $1.95(p=0.052)$ & $4.83(2.9)$ &.. &.. \\
\hline$\geq 75$ & $4.69(5 \cdot 7)$ & $4 \cdot 4(3 \cdot 9)$ & $0 \cdot 29(p=0 \cdot 61)$ & $3 \cdot 26(4 \cdot 4)$ & $3.05(3.4)$ & $0.37(p=0 \cdot 71)$ & $5 \cdot 22(3 \cdot 1)$ &.. &.. \\
\hline
\end{tabular}

${ }^{7}$ PHQ-9, the 9-item Patient Health Questionnaire; ${ }^{19}$ GAD-7, the 7-item Generalized Anxiety Disorder Scale ${ }^{20}$ PSS-4, the 4-item Perceived Stress Scale. ${ }^{21}$ Published population normative data for PHQ- $9^{23}$, GAD- ${ }^{25}$, PSS- $4^{24}$.

$* * * * p<0 \cdot 0001, * * * p<0 \cdot 001, * * p<0.01, * p<0.05$ 
Table 3: Prevalence of depressive and anxiety cases ${ }^{\dagger}$

\begin{tabular}{|c|c|c|c|c|c|c|c|}
\hline & \multirow[b]{2}{*}{ Categories } & \multicolumn{2}{|c|}{ Whole sample } & \multicolumn{2}{|c|}{ Male } & \multicolumn{2}{|c|}{ Female } \\
\hline & & & $\%$ & $\mathbf{n}$ & $\%$ & $\mathbf{n}$ & $\%$ \\
\hline \multirow[t]{5}{*}{ Depression (PHQ-9 $\left.{ }^{5}\right)$} & No-Minimal Depression (0-4) & 1125 & $36 \cdot 3$ & 230 & $48 \cdot 3$ & 894 & $34 \cdot 1$ \\
\hline & Mild Depression (5-9) & 994 & $32 \cdot 1$ & 125 & $26 \cdot 3$ & 868 & $33 \cdot 2$ \\
\hline & Moderate Depression (10-14) & 525 & $17 \cdot 0$ & 64 & $13 \cdot 4$ & 461 & $17 \cdot 6$ \\
\hline & Moderately Severe Depression (15-19) & 276 & 8.9 & 35 & $7 \cdot 4$ & 241 & $9 \cdot 2$ \\
\hline & Severe Depression (20-27) & 177 & $5 \cdot 7$ & 22 & $4 \cdot 6$ & 154 & $5 \cdot 9$ \\
\hline \multirow[t]{4}{*}{ Anxiety $\left(\right.$ GAD- $\left.7^{\ddagger}\right)$} & No-Minimal Anxiety (0-4) & 1344 & $43 \cdot 4$ & 276 & $58 \cdot 0$ & 1066 & $40 \cdot 7$ \\
\hline & Mild Anxiety (5-9) & 947 & $30 \cdot 6$ & 108 & 22.7 & 839 & $32 \cdot 0$ \\
\hline & Moderate Anxiety (10-14) & 430 & $13 \cdot 9$ & 44 & $9 \cdot 2$ & 386 & 14.7 \\
\hline & Severe Anxiety (15-21) & 376 & $12 \cdot 1$ & 48 & $10 \cdot 1$ & 327 & $12 \cdot 5$ \\
\hline
\end{tabular}

Cut-offs for categories in line with published guidelines for PHQ-9 ${ }^{23}$ and GAD-7. ${ }^{25}$

${ }^{\ddagger}$ PHQ-9, the 9-item Patient Health Questionnaire; ${ }^{19}$ GAD-7, the 7-item Generalized Anxiety Disorder Scale. ${ }^{20}$ 
Individuals at greatest risk of mental health problems: associations with age, gender, ethnicity, living alone and key-worker status

When non-modifiable explanatory variables were included in a multivariable model (Table 4), we observed that for depression (square-root transformed scores), being younger ( $\mathrm{B}=-0 \cdot 28,95 \% \mathrm{CI}:-0 \cdot 31,-0 \cdot 25$ per decade), female $(B=0 \cdot 36,95 \%$ CI: $0 \cdot 25,0 \cdot 47)$ and living alone $(B=0 \cdot 34,95 \%$ CI: $0 \cdot 25,0 \cdot 47)$ were all independently significantly associated with greater levels of depression. This model accounted for approximately $13 \%$ of the variance in depression scores. These results were replicated when considering depression as a binary outcome (i.e., cases requiring high intensity intervention versus not) with females having a $49 \%$ increased odds of depression and living alone associated with a $55 \%$ increase.

Table 4: Regression models showing associations between non-modifiable explanatory variables and depression scores

\begin{tabular}{|c|c|c|c|c|c|}
\hline & B & 95\% CI Lower & 95\% CI Upper & $\beta$ & $p$ \\
\hline \multicolumn{6}{|l|}{ PHQ-9 Total Score $^{\text {a }}$} \\
\hline Age (per decade) & $-0 \cdot 28$ & $-0 \cdot 31$ & $-0 \cdot 25$ & $-0 \cdot 35$ & $<\cdot 0001 * * * *$ \\
\hline Female & $0 \cdot 36$ & $0 \cdot 25$ & $0 \cdot 47$ & $0 \cdot 11$ & $<\cdot 0001 * * * *$ \\
\hline Live alone & $0 \cdot 34$ & $0 \cdot 22$ & $0 \cdot 46$ & 0.09 & $<\cdot 0001^{* * * *}$ \\
\hline BAME background & 0.03 & $-0 \cdot 11$ & $0 \cdot 17$ & $0 \cdot 01$ & .64 \\
\hline Key-worker & 0.06 & $-0 \cdot 02$ & $0 \cdot 14$ & $0 \cdot 02$ & $\cdot 15$ \\
\hline \multicolumn{6}{|l|}{ Adjusted $R^{2}=0 \cdot 13, n=3090$} \\
\hline & Odds Ratio & 95\% CI Lower & 95\% CI Upper & $\beta$ & $p$ \\
\hline \multicolumn{6}{|l|}{ PHQ-9 "Cases" b } \\
\hline Age (per decade) & $0 \cdot 67$ & 0.63 & 0.71 & $-1 \cdot 30$ & $<\cdot 0001 * * * *$ \\
\hline Female & 1.49 & $1 \cdot 18$ & 1.89 & $0 \cdot 31$ & $.00079 * * *$ \\
\hline Live alone & 1.55 & $1 \cdot 23$ & 1.97 & $0 \cdot 32$ & $.00025^{* * *}$ \\
\hline BAME background & $1 \cdot 14$ & $0 \cdot 88$ & $1 \cdot 48$ & $0 \cdot 08$ & 32 \\
\hline Key-worker & $1 \cdot 14$ & 0.97 & $1 \cdot 33$ & $0 \cdot 14$ & .11 \\
\hline Pseudo $R^{2}=0 \cdot 06, n=3090$ & & & & & \\
\hline
\end{tabular}

$* * * * p<0 \cdot 0001, * * * p<0 \cdot 001, * * p<0 \cdot 01, * p<0 \cdot 05$

${ }^{a}$ A square-root transformation was applied to the dependent variable.

b a "case" is defined as a PHQ-9 score greater than or equal to 10, at which level someone would qualify for high intensity psychological support in the NHS.

For anxiety (square-root transformed scores) being younger ( $\mathrm{B}=-0 \cdot 24,95 \% \mathrm{CI}$ : $-0 \cdot 27,-0 \cdot 22$ per decade) and female $(B=0 \cdot 43,95 \%$ CI: $0 \cdot 32,0 \cdot 55)$ were independently significantly associated with greater levels of anxiety (Table 5). This model accounted for approximately $11 \%$ of the variance and these results were replicated when considering anxiety as a binary outcome (i.e., cases requiring high intensity intervention versus not). 
medRxiv preprint doi: https://doi.org/10.1101/2020.05.14.20102012; this version posted May 19, 2020. The copyright holder for this preprint (which was not certified by peer review) is the author/funder, who has granted medRxiv a license to display the preprint in perpetuity.

It is made available under a CC-BY 4.0 International license .

Table 5: Regression models showing associations between non-modifiable explanatory variables and anxiety scores

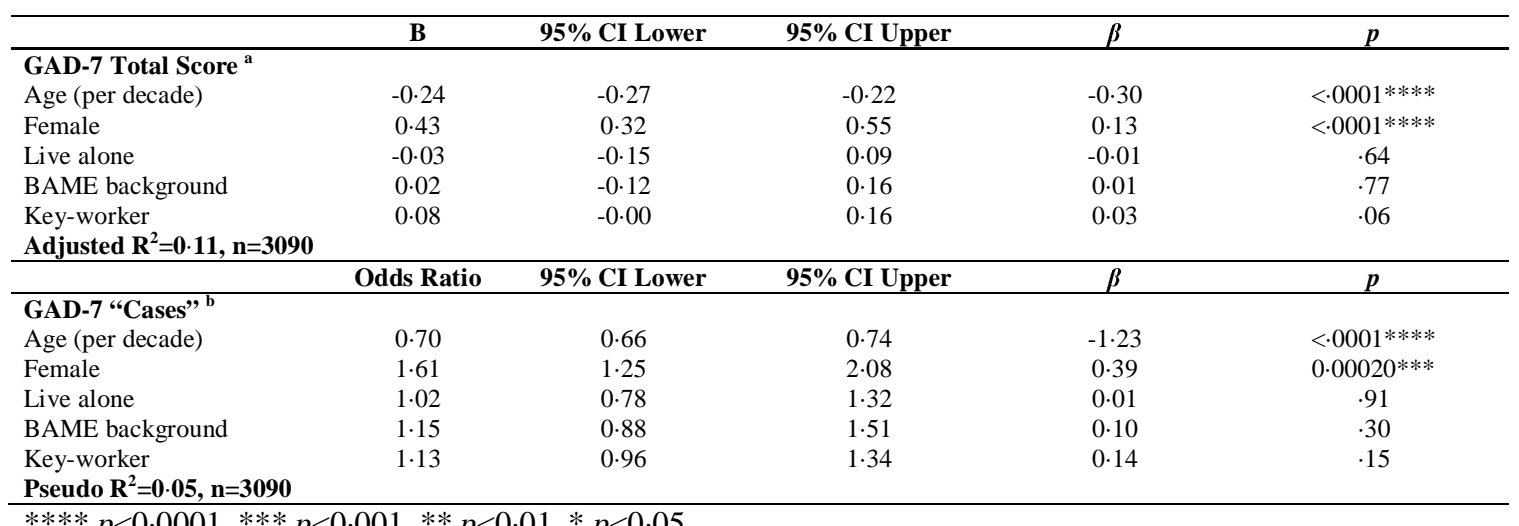

$* * * * p<0 \cdot 0001, * * * p<0 \cdot 001, * * p<0 \cdot 01, * p<0 \cdot 05$

${ }^{a}$ A square-root transformation was applied to the dependent variable.

b a "case" is defined as a GAD-7 score greater than or equal to 10, at which level someone would qualify for high intensity psychological support in the NHS.

For stress, being younger $(\mathrm{B}=-0 \cdot 54,95 \% \mathrm{CI}:-0 \cdot 61,-0 \cdot 46$ per decade $)$, female $(\mathrm{B}=0 \cdot 78,95 \% \mathrm{CI}: 0 \cdot 46,1 \cdot 09)$, living alone $(B=0 \cdot 48,95 \% \mathrm{CI}: 0 \cdot 15,0 \cdot 82)$, being from a BAME background ( $\mathrm{B}=0 \cdot 45,95 \% \mathrm{CI}: 0 \cdot 07,0 \cdot 84)$, were all independently significantly associated with greater stress; while being a key-worker was independently significantly associated with a lower stress $(B=-0 \cdot 24,95 \% \mathrm{CI}$ : $-0 \cdot 47,-0 \cdot 02)$. Together the model accounted for approximately $7 \%$ of the variance in stress scores (Table 6).

Table 6: Regression model showing associations between non-modifiable explanatory variables and stress scores

\begin{tabular}{|c|c|c|c|c|c|}
\hline & B & 95\% CI Lower & 95\% CI Upper & $\beta$ & $p$ \\
\hline \multicolumn{6}{|l|}{ PSS-4 Total Score } \\
\hline Age (per decade) & -0.54 & $-0 \cdot 61$ & $-0 \cdot 46$ & -0.25 & $<.0001 * * * *$ \\
\hline Female & 0.78 & 0.46 & 1.09 & 0.09 & $<.0001 * * * *$ \\
\hline Live alone & $0 \cdot 48$ & $0 \cdot 15$ & $0 \cdot 82$ & $0 \cdot 05$ & $0 \cdot 0049 * *$ \\
\hline BAME background & $0 \cdot 45$ & $0 \cdot 07$ & $0 \cdot 84$ & $0 \cdot 04$ & $0 \cdot 022 *$ \\
\hline Key-worker & $-0 \cdot 24$ & $-0 \cdot 47$ & $-0 \cdot 02$ & $-0 \cdot 04$ & $0.033^{*}$ \\
\hline Adjusted $R^{2}=0 \cdot 07$, & & & & & \\
\hline
\end{tabular}

Individuals at greatest risk of mental health problems: associations with perceived risk of COVID-19, perceived loneliness, COVID-19 worry and positive mood

Table 7 shows levels of modifiable explanatory variables (perceived risk, perceived loneliness, COVID-19 worry, and positive mood) across the whole sample, as well as by gender and age-groups. 
Table 7: Loneliness, worry about COVID-19, perceived risk of COVID-19, and positive mood

\begin{tabular}{|c|c|c|c|c|c|c|c|c|c|c|}
\hline & \multirow[b]{2}{*}{ Whole sample } & \multicolumn{2}{|c|}{ Gender } & \multicolumn{7}{|c|}{ Age groups (years) } \\
\hline & & Male & Female & $18-24$ & $25-34$ & $35-44$ & $45-54$ & $55-64$ & $65-74$ & $\geq 75$ \\
\hline \multicolumn{11}{|l|}{ Loneliness } \\
\hline Mean (SD) & $3.86(2 \cdot 7)$ & $3.56(2 \cdot 7)$ & $3.91(2.7)$ & $5 \cdot 36(2 \cdot 7)$ & $4 \cdot 36(2 \cdot 7)$ & $3 \cdot 76(2 \cdot 7)$ & $3 \cdot 62(2 \cdot 8)$ & $3.47(2.7)$ & $2 \cdot 70(2 \cdot 1)$ & $2.71(2.4)$ \\
\hline \multicolumn{11}{|l|}{ Positive mood } \\
\hline Mean (SD) & $18.99(5 \cdot 1)$ & $19 \cdot 76(5 \cdot 1)$ & $18 \cdot 85(5 \cdot 0)$ & $17 \cdot 67(4 \cdot 9)$ & $18 \cdot 82(5 \cdot 1)$ & $18 \cdot 67(5 \cdot 0)$ & $18 \cdot 92(5 \cdot 1)$ & $19 \cdot 38(5 \cdot 0)$ & $20 \cdot 72(4 \cdot 6)$ & $21 \cdot 56(5 \cdot 2)$ \\
\hline \multicolumn{11}{|l|}{$\begin{array}{l}\text { Perceived risk of } \\
\text { COVID-19 }\end{array}$} \\
\hline Mean (SD) & $4.75(2 \cdot 2)$ & $4 \cdot 46(2 \cdot 2)$ & $4 \cdot 80(2 \cdot 2)$ & $4 \cdot 10(2 \cdot 0)$ & $4.92(2 \cdot 2)$ & $5 \cdot 15(2 \cdot 2)$ & $5(2 \cdot 2)$ & $4 \cdot 78(2 \cdot 3)$ & $4 \cdot 21(2 \cdot 1)$ & $3 \cdot 31(1.9)$ \\
\hline \multicolumn{11}{|l|}{$\begin{array}{l}\text { Worry about COVID- } \\
19\end{array}$} \\
\hline No worry $(\mathrm{n}, \%)$ & $512(16 \cdot 5 \%)$ & $105(22 \cdot 1 \%)$ & $406(15 \cdot 5 \%)$ & $105(28.9 \%)$ & $108(20 \cdot 5 \%)$ & $91(14 \cdot 3 \%)$ & $91(13 \cdot 3 \%)$ & $65(11.4 \%)$ & $39(15 \cdot 4 \%)$ & $13(21 \cdot 0 \%)$ \\
\hline Occasional worry $(\mathrm{n}, \%)$ & $2050(66 \cdot 2 \%)$ & $318(66 \cdot 8 \%)$ & $1731(66 \cdot 1 \%)$ & $208(57 \cdot 3 \%)$ & $320(60 \cdot 6 \%)$ & $427(67 \cdot 2 \%)$ & $466(67 \cdot 8 \%)$ & $\begin{array}{c}396 \\
(69 \cdot 7 \%)\end{array}$ & $\begin{array}{c}188 \\
(74 \cdot 0 \%)\end{array}$ & $45(72.6 \%)$ \\
\hline Much worry (n, \%) & $413(13 \cdot 3 \%)$ & $40(8 \cdot 4 \%)$ & $373(14 \cdot 3 \%)$ & $39(10 \cdot 7 \%)$ & $77(14 \cdot 6 \%)$ & $91(14.3 \%)$ & $94(13.7 \%)$ & $85(15.0 \%)$ & $24(9 \cdot 5 \%)$ & $3(4.8 \%)$ \\
\hline Most worry (n, \%) & $122(3.9 \%)$ & $13(2 \cdot 7 \%)$ & $108(4 \cdot 1 \%)$ & $11(3 \cdot 0 \%)$ & $23(4.4 \%)$ & $26(4 \cdot 1 \%)$ & $36(5 \cdot 2 \%)$ & $22(3.9 \%)$ & $3(1.2 \%)$ & $1(1.6 \%)$ \\
\hline
\end{tabular}


When modifiable explanatory variables were added into the multivariable model for depression: this revealed that greater perceived loneliness $(\mathrm{B}=0 \cdot 10,95 \% \mathrm{CI}: 0 \cdot 09,0 \cdot 12)$, lower positive $\operatorname{mood}(\mathrm{B}=-0 \cdot 12,95 \% \mathrm{CI}:-0 \cdot 12$, $0 \cdot 11$ ) and greater than occasional worry about getting COVID-19 (much of time: $\mathrm{B}=0 \cdot 28,95 \% \mathrm{CI}: 0 \cdot 18,0 \cdot 38$; most of time: $\mathrm{B}=0 \cdot 32,95 \% \mathrm{CI}: 0 \cdot 13,0 \cdot 50)$, were all independently and significantly associated with greater levels of depression, in addition to age and gender. The model accounted for approximately $56 \%$ of the variance in depression scores. While perceived risk of COVID-19 was not statistically significant, in sensitivity analyses where large residuals were excluded $(<-3 />3)$ this became statistically significant $(\mathrm{B}=0 \cdot 02,95 \% \mathrm{CI}: 0 \cdot 00,0 \cdot 03)$. These results were largely replicated when considering depression as a binary outcome although gender was no longer statistically significant (Table 8).

Table 8: Regression models showing associations between modifiable explanatory variables and depression scores

\begin{tabular}{|c|c|c|c|c|c|}
\hline & $\mathbf{B}$ & 95\% CI Lower & 95\% CI Upper & $\beta$ & $p$ \\
\hline \multicolumn{6}{|l|}{ PHQ-9 Total Score $^{\text {a }}$} \\
\hline Age (per decade) & $-0 \cdot 18$ & $-0 \cdot 20$ & $-0 \cdot 15$ & $-0 \cdot 22$ & $<\cdot 0001 * * * *$ \\
\hline Female & $0 \cdot 18$ & $0 \cdot 09$ & $0 \cdot 27$ & $0 \cdot 05$ & $<\cdot 0001 * * * *$ \\
\hline Live alone & $0 \cdot 02$ & $-0 \cdot 08$ & $0 \cdot 12$ & $0 \cdot 01$ & 0.71 \\
\hline BAME background & $-0 \cdot 03$ & $-0 \cdot 14$ & $0 \cdot 08$ & $-0 \cdot 01$ & $0 \cdot 61$ \\
\hline Key-worker & $0 \cdot 01$ & $-0 \cdot 06$ & $0 \cdot 08$ & $0 \cdot 00$ & $0 \cdot 84$ \\
\hline Perceived loneliness (per unit) & $0 \cdot 10$ & 0.09 & $0 \cdot 12$ & $0 \cdot 23$ & $<\cdot 0001 * * * *$ \\
\hline Positive mood (per unit) & $-0 \cdot 12$ & $-0 \cdot 12$ & $-0 \cdot 11$ & $-0 \cdot 48$ & $<\cdot 0001 * * * *$ \\
\hline \multicolumn{6}{|l|}{ COVID-19 worry $^{\mathrm{b}}$} \\
\hline No worry & $-0 \cdot 01$ & $-0 \cdot 10$ & $0 \cdot 09$ & $-0 \cdot 00$ & $0 \cdot 89$ \\
\hline Much of time & $0 \cdot 28$ & $0 \cdot 18$ & $0 \cdot 38$ & $0 \cdot 08$ & $<.0001 * * * *$ \\
\hline Most of time & $0 \cdot 32$ & $0 \cdot 13$ & $0 \cdot 50$ & $0 \cdot 05$ & $0 \cdot 00067 * * *$ \\
\hline $\begin{array}{l}\text { Perceived risk of COVID-19 (per } \\
\text { unit) }\end{array}$ & $0 \cdot 01$ & $-0 \cdot 00$ & 0.03 & $0 \cdot 02$ & $0 \cdot 13$ \\
\hline \multicolumn{6}{|l|}{ Adjusted $R^{2}=0 \cdot 56, n=2494$} \\
\hline & Odds Ratio & 95\% CI Lower & 95\% CI Upper & $\beta$ & $p$ \\
\hline \multicolumn{6}{|l|}{ PHQ-9 "Cases" c } \\
\hline Age (per decade) & $0 \cdot 68$ & 0.63 & $0 \cdot 74$ & $-1 \cdot 30$ & $<\cdot 0001 * * * *$ \\
\hline Female & $1 \cdot 06$ & $0 \cdot 76$ & 1.47 & $0 \cdot 04$ & $0 \cdot 75$ \\
\hline Live alone & $0 \cdot 88$ & $0 \cdot 61$ & $1 \cdot 25$ & $-0 \cdot 10$ & $0 \cdot 46$ \\
\hline BAME background & $0 \cdot 95$ & $0 \cdot 65$ & $1 \cdot 39$ & $-0 \cdot 03$ & 0.79 \\
\hline Key-worker & 1.07 & $0 \cdot 85$ & $1 \cdot 36$ & $0 \cdot 08$ & 0.57 \\
\hline Perceived loneliness (per unit) & $1 \cdot 22$ & $1 \cdot 17$ & $1 \cdot 28$ & $1 \cdot 20$ & $<.0001 * * * *$ \\
\hline Positive mood (per unit) & 0.76 & 0.74 & 0.79 & $-3 \cdot 02$ & $<.0001 * * * *$ \\
\hline \multicolumn{6}{|l|}{ COVID-19 worry $^{b}$} \\
\hline No worry & $1 \cdot 00$ & 0.71 & 1.41 & $0 \cdot 00$ & $0 \cdot 98$ \\
\hline Much of time & $1 \cdot 74$ & $1 \cdot 28$ & $2 \cdot 36$ & $0 \cdot 41$ & 0.00037 *** \\
\hline Most of time & $2 \cdot 08$ & $1 \cdot 17$ & $3 \cdot 72$ & $0 \cdot 30$ & $0.013^{*}$ \\
\hline $\begin{array}{l}\text { Perceived risk of COVID-19 (per } \\
\text { unit) }\end{array}$ & $1 \cdot 04$ & 0.98 & 1.09 & $0 \cdot 17$ & $0 \cdot 23$ \\
\hline \multicolumn{6}{|l|}{ Pseudo $R^{2}=0 \cdot 35, n=2494$} \\
\hline \multicolumn{6}{|c|}{$* * * * p<0.0001, * * * p<0.001, * * p<0.01, * p<0.05$} \\
\hline \multicolumn{6}{|c|}{${ }^{a}$ A square-root transformation was applied to the dependent variable. } \\
\hline \multicolumn{6}{|c|}{ b Comparison reference group "I occasionally worry about getting COVID-19”. } \\
\hline \multicolumn{6}{|c|}{ " a "case" is defined as a PHQ-9 score greater than or equal to 10 , at which level someone would qualify for } \\
\hline
\end{tabular}

For anxiety, the model revealed that greater perceived loneliness $(\mathrm{B}=0 \cdot 06,95 \% \mathrm{CI}: 0 \cdot 04,0 \cdot 07)$, lower positive mood $(B=-0 \cdot 12,95 \% \mathrm{CI}:-0 \cdot 13,-0 \cdot 11)$ and greater perceived risk of COVID-19 (B=0.04, 95\% CI: 0.02, 0.05) were all independently and significantly associated with greater anxiety, in addition to the non-modifiable factors of being younger, female and living alone. Further, those participants who experienced greater than occasional worry about getting COVID-19 were significantly more likely to have higher levels of anxiety (much of time: $\mathrm{B}=0 \cdot 58,95 \% \mathrm{CI}: 0 \cdot 47,0 \cdot 68$; most of time: $\mathrm{B}=0 \cdot 87,95 \% \mathrm{CI}: 0 \cdot 68,1 \cdot 06)$; with those who did not worry at all about getting COVID-19 being likely to have lower anxiety $(\mathrm{B}=-0 \cdot 19,95 \% \mathrm{CI}$ : $-0 \cdot 28,-0 \cdot 09)$. The model accounted for approximately $54 \%$ of the variance in anxiety scores. These results were largely replicated when considering anxiety as a binary outcome, although gender and not worrying at all about getting COVID-19 were no longer statistically significant (Table 9). 
medRxiv preprint doi: https://doi.org/10.1101/2020.05.14.20102012; this version posted May 19, 2020. The copyright holder for this preprint (which was not certified by peer review) is the author/funder, who has granted medRxiv a license to display the preprint in perpetuity.

It is made available under a CC-BY 4.0 International license .

Table 9: Regression models showing associations between modifiable explanatory variables and anxiety

\begin{tabular}{|c|c|c|c|c|c|}
\hline & B & 95\% CI Lower & 95\% CI Upper & $\beta$ & $p$ \\
\hline \multicolumn{6}{|l|}{ GAD-7 Total Score $^{\text {a }}$} \\
\hline Age (per decade) & $-0 \cdot 16$ & $-0 \cdot 18$ & $-0 \cdot 13$ & $-0 \cdot 19$ & $<.0001 * * * *$ \\
\hline Female & $0 \cdot 25$ & $0 \cdot 15$ & $0 \cdot 34$ & $0 \cdot 07$ & $<\cdot 0001 * * * *$ \\
\hline Live alone & $-0 \cdot 25$ & $-0 \cdot 35$ & $-0 \cdot 15$ & $-0 \cdot 07$ & $<\cdot 0001 * * * *$ \\
\hline BAME background & $-0 \cdot 08$ & $-0 \cdot 20$ & 0.03 & $-0 \cdot 02$ & $0 \cdot 17$ \\
\hline Key-worker & $-0 \cdot 04$ & $-0 \cdot 11$ & 0.03 & $-0 \cdot 02$ & $0 \cdot 27$ \\
\hline Perceived loneliness (per unit) & $0 \cdot 06$ & $0 \cdot 04$ & $0 \cdot 07$ & $0 \cdot 12$ & $<.0001 * * * *$ \\
\hline Positive mood (per unit) & $-0 \cdot 12$ & $-0 \cdot 13$ & $-0 \cdot 11$ & $-0 \cdot 48$ & $<.0001 * * * *$ \\
\hline \multicolumn{6}{|l|}{ COVID-19 worry ${ }^{\mathrm{b}}$} \\
\hline No worry & $-0 \cdot 19$ & $-0 \cdot 28$ & $-0 \cdot 09$ & $-0 \cdot 05$ & $0.00015^{* * *} *$ \\
\hline Much of time & $0 \cdot 58$ & 0.47 & 0.68 & $0 \cdot 16$ & $<.0001 * * * *$ \\
\hline Most of time & $0 \cdot 87$ & $0 \cdot 68$ & 1.06 & $0 \cdot 13$ & $<.0001 * * * *$ \\
\hline $\begin{array}{l}\text { Perceived risk of COVID-19 (per } \\
\text { unit) }\end{array}$ & $0 \cdot 04$ & $0 \cdot 02$ & $0 \cdot 05$ & $0 \cdot 06$ & $<\cdot 0001 * * * *$ \\
\hline \multicolumn{6}{|l|}{ Adjusted $R^{2}=\cdot 54, n=2494$} \\
\hline & Odds Ratio & 95\% CI Lower & 95\% CI Upper & $\beta$ & $p$ \\
\hline \multicolumn{6}{|l|}{ GAD-7 “Cases" c } \\
\hline Age (per decade) & $0 \cdot 69$ & $0 \cdot 63$ & $0 \cdot 75$ & $-1 \cdot 34$ & $<.0001 * * * *$ \\
\hline Female & $1 \cdot 17$ & $0 \cdot 82$ & 1.67 & $0 \cdot 14$ & $0 \cdot 37$ \\
\hline Live alone & $0 \cdot 67$ & 0.46 & 0.99 & $-0 \cdot 32$ & $0 \cdot 044^{*}$ \\
\hline BAME background & $0 \cdot 96$ & $0 \cdot 65$ & 1.43 & $-0 \cdot 03$ & $0 \cdot 85$ \\
\hline Key-worker & $0 \cdot 90$ & $0 \cdot 70$ & $1 \cdot 15$ & $-0 \cdot 13$ & $0 \cdot 40$ \\
\hline Perceived loneliness (per unit) & $1 \cdot 11$ & 1.06 & $1 \cdot 17$ & $0 \cdot 67$ & $<.0001 * * * *$ \\
\hline Positive mood (per unit) & $0 \cdot 77$ & $0 \cdot 75$ & $0 \cdot 80$ & $-3 \cdot 07$ & $<.0001 * * * *$ \\
\hline \multicolumn{6}{|l|}{ COVID-19 worr $y^{\mathrm{b}}$} \\
\hline No worry & $0 \cdot 75$ & $0 \cdot 52$ & 1.09 & $-0 \cdot 24$ & $0 \cdot 14$ \\
\hline Much of time & $3 \cdot 86$ & $2 \cdot 86$ & $5 \cdot 22$ & 1.06 & $<.0001 * * * *$ \\
\hline Most of time & 11.57 & $5 \cdot 88$ & $22 \cdot 77$ & 1.06 & $<.0001 * * * *$ \\
\hline $\begin{array}{l}\text { Perceived risk of COVID-19 (per } \\
\text { unit) } \\
\text { Pseudo } \mathbf{R}^{\mathbf{2}}=\mathbf{0} \cdot \mathbf{3 6}, \mathbf{n}=\mathbf{2 4 9 4}\end{array}$ & $1 \cdot 07$ & $1 \cdot 01$ & $1 \cdot 14$ & $0 \cdot 35$ & $0 \cdot 024^{*}$ \\
\hline \multicolumn{6}{|c|}{$* * * * p<0 \cdot 0001, * * * p<0 \cdot 001, * * p<0 \cdot 01, * p<0 \cdot 05$} \\
\hline \multicolumn{6}{|c|}{${ }^{a}$ A square-root transformation was applied to the dependent variable. } \\
\hline \multicolumn{6}{|c|}{ b Comparison reference group "I occasionally worry about getting COVID-19". } \\
\hline \multicolumn{6}{|c|}{ "a "case" is defined as a GAD-7 score greater than or equal to 10 , at which level someone would qualify for } \\
\hline
\end{tabular}

The multivariable model for stress scores showed that greater perceived loneliness $(\mathrm{B}=0 \cdot 19,95 \% \mathrm{CI}: 0 \cdot 15$, $0 \cdot 23)$, lower positive mood $(\mathrm{B}=-0 \cdot 38,95 \% \mathrm{CI}:-0 \cdot 40,-0 \cdot 36)$, greater than occasional worry about getting COVID-19 (much of time: $\mathrm{B}=0 \cdot 37,95 \% \mathrm{CI}: 0 \cdot 10,0 \cdot 63$; most of time: $\mathrm{B}=1 \cdot 02,95 \% \mathrm{CI}: 0 \cdot 54,1 \cdot 50$ ), and greater perceived risk of getting COVID-19 (B=0.06, 95\% CI:0.02, 0.11) were all independently and significantly associated with greater stress, in addition to being younger, female, living alone and not being a key-worker. In robustness analyses, when removing large residuals $(<-3$ or $>3)$ having a BAME background was also a statistically significant independent predictor $(B=0 \cdot 29,95 \% \mathrm{CI}: 0 \cdot 00,0 \cdot 58)$. This model accounted for approximately $57 \%$ of the variance in stress scores (Table 10). 
Table 10: Regression model showing associations between modifiable explanatory variables and stress scores

\begin{tabular}{|c|c|c|c|c|c|}
\hline & B & 95\% CI Lower & 95\% CI Upper & $\beta$ & $p$ \\
\hline \multicolumn{6}{|l|}{ PSS-4 Total Score } \\
\hline Age (per decade) & $-0 \cdot 24$ & $-0 \cdot 30$ & $-0 \cdot 18$ & $-0 \cdot 11$ & $<.0001 * * * *$ \\
\hline Female & $0 \cdot 35$ & $0 \cdot 12$ & 0.59 & $0 \cdot 04$ & $0.0035^{* *}$ \\
\hline Live alone & -0.41 & $-0 \cdot 67$ & $-0 \cdot 14$ & -0.04 & $0.0025^{* *}$ \\
\hline BAME background & $0 \cdot 26$ & -0.04 & 0.55 & $0 \cdot 02$ & 0.088 \\
\hline Key-worker & $-0 \cdot 40$ & $-0 \cdot 58$ & $-0 \cdot 21$ & -0.06 & $<.0001 * * * *$ \\
\hline Perceived loneliness (per unit) & $0 \cdot 19$ & $0 \cdot 15$ & $0 \cdot 23$ & $0 \cdot 15$ & $<.0001 * * * *$ \\
\hline Positive mood (per unit) & -0.38 & $-0 \cdot 40$ & $-0 \cdot 36$ & $-0 \cdot 60$ & $<.0001$ ***** \\
\hline \multicolumn{6}{|l|}{ COVID-19 worry ${ }^{\mathrm{a}}$} \\
\hline No worry & $-0 \cdot 05$ & $-0 \cdot 30$ & $0 \cdot 19$ & $-0 \cdot 01$ & 0.67 \\
\hline Much of time & $0 \cdot 37$ & $0 \cdot 10$ & 0.63 & $0 \cdot 04$ & $0 \cdot 0068 * *$ \\
\hline Most of time & 1.02 & 0.54 & 1.50 & $0 \cdot 06$ & $<.0001 * * * *$ \\
\hline $\begin{array}{l}\text { Perceived risk of COVID-19 (per } \\
\text { unit) }\end{array}$ & 0.06 & 0.02 & $0 \cdot 11$ & $0 \cdot 04$ & $0 \cdot 0037 * *$ \\
\hline Adjusted $R^{2}=\cdot 57, n=2494$ & & & & & \\
\hline
\end{tabular}

\section{Discussion}

We report findings from a community cohort study established in the UK to examine the mental health consequences of the COVID-19 pandemic. Our results pertain to the experiences of people within the first four to six weeks of social distancing measures being introduced, and focus on self-reported depression, anxiety and stress scores. The findings indicated that mean levels of depression, anxiety and stress significantly exceeded recent population norms. ${ }^{23-25}$ Models examining the relationship between these mental health outcomes and nonmodifiable explanatory factors accounted for only a modest proportion of the variance (7-13\%). Increased depression was associated with being younger, female and living alone; increased anxiety was associated with being younger and female; and increased stress was associated with being younger, female, living alone, being from a BAME background and not being a keyworker. In contrast, when we added the hypothesised modifiable variables into our multivariable models we observed that they accounted for a much larger proportion of the variance (54-57\%) with significant independent effects emerging for positive mood, perceived loneliness and worry about getting COVID-19 for all three outcomes, as well as perceived risk of COVID-19 emerging as significant for anxiety and stress.

These findings highlight a number of issues worthy of discussion. First, both mean scores and measures of caseness indicate that the COVID-19 pandemic is having widespread and deleterious effects on the emotional wellbeing of people in the UK. This is true for depression, generalised anxiety disorder and stress and is in keeping with observations from other countries. ${ }^{2,3}$ Indeed, the proportion of participants who would require intensive support for depression and anxiety in the NHS does not compare favourably with recent historical estimates of the prevalence of mental health problems in the UK. For example, the 2014 ONS report on adult psychiatric morbidity reported a prevalence of $17 \%$ for six different common mental disorders. ${ }^{27}$ The prevalence of depression alone in the context of this pandemic is almost double this.

Second, the non-modifiable explanatory variables significantly associated with all three of our mental health outcomes were being younger and being female. These findings are consistent with unpublished data from another UK community cohort recruited during the COVID-19 pandemic with a similar gender profile to our own, ${ }^{28}$ suggesting that these groups may be the most in need of intervention. Although this runs counter to our hypothesis that the greatest psychological morbidity would be observed in individuals at greatest risk of COVID-19, it is consistent with previous work which has shown that individual's perceptions of disease risk are often poorly related to actual risk. ${ }^{29}$ Alternatively, the results may reflect the fact that the pandemic has resulted in a panoply of challenges to mental health that go beyond the disease itself. It could be hypothesised, for example, that some of the more immediate consequences such as unemployment, financial concerns and increased domestic violence would disproportionately affect younger people and women and this may explain our findings.

A third, and related issue, is that although being younger and female were consistently associated with poorer mental health, the relationship was modest, accounting for, at best, $13 \%$ of the variance. In contrast, the 
modifiable explanatory measures when added to the multivariable models accounted for $52-57 \%$ of the variance. These findings are encouraging as they suggest that there is considerable potential for us to develop interventions to mitigate the mental health effects of the pandemic. The measures of perceived loneliness, positive mood and worry about getting COVID-19 were strongly associated with all three outcomes and thus would be appropriate cognitions to be targeted in future interventions. ${ }^{30}$

A further issue concerns the effects of the pandemic beyond mental health. It is well known that when negative mood states persist over time they result in the dysregulation of physiological systems involved in the regulation of the immune system. ${ }^{31}$ Thus, there exists significant potential for the psychological harm inflicted by the pandemic to translate into physical harm. This could include an increased susceptibility to the virus, worse outcomes if infected, or indeed poorer responses to vaccinations in the future. ${ }^{32}$ Studies providing longitudinal data on the prevalence of psychological morbidity and appropriate biomarkers (e.g., cortisol) will be required to determine whether the risks to physical health go beyond the hypothetical.

Finally, we would like to acknowledge several limitations. These include the cross-sectional design of the work which impedes an analysis of cause and effect; the limited generalisability of our cohort inflicted by the selfselected community cohort design and the absence of information on pre-existing mental health conditions which are likely to impact on the severity and prevalence of psychological morbidity. ${ }^{1}$ Nonetheless, we are among the first to provide evidence from a large cohort on the mental health impact of the COVID-19 pandemic on people in the UK; to identify groups who may be at particular risk, as well as potential targets for therapeutic intervention. 


\begin{abstract}
Author contributions
$\mathrm{Ru}$ Jia: study design, coordination and management of recruitment, preparation, analysis and interpretation of data and preparation of manuscript.
\end{abstract}

Kieran Ayling: study design, coordination and management of recruitment, preparation, analysis and interpretation of data and preparation of manuscript.

Trudie Chalder: study design, analysis and interpretation of data and preparation of manuscript

Adam Massey: study design, coordination and management of recruitment, preparation, analysis and interpretation of data and preparation of manuscript.

Elizabeth Broadbent: study design, interpretation of data and preparation of manuscript

Carol Coupland: study design, analysis and interpretation of data and preparation of manuscript

Kavita Vedhara: research lead, study design, coordination and management of recruitment, preparation, analysis and interpretation of data and preparation of manuscript.

As corresponding author, KV had access to all the data in the study and had final responsibility for the decision to submit for publication

\title{
References
}

1. Holmes EA, O'Connor RC, Perry VH, et al. Multidisciplinary research priorities for the COVID-19 pandemic: a call for action for mental health science [published online ahead of print, 2020 Apr 15]. Lancet Psychiatry. 2020;S2215-0366(20)30168-1. doi:10.1016/S2215-0366(20)30168-1

2. Liu S, Yang L, Zhang C et al. Online mental health services in China during the COVID $\square 19$ outbreak. Lancet Psychiatry 2020; 18:e17-e18 doi.org/10.1016/S2215-0366(20)30077-8

3. Tian F, Li H, Tian S, Yang J, Shao J, Tian C. Psychological symptoms of ordinary Chinese citizens based on SCL-90 during the level I emergency response to COVID-19 [published online ahead of print, 2020 Apr 11]. Psychiatry Res. 2020; 288-112992. doi:10.1016/j.psychres.2020.112992

4. Jeong H, Yim HW, Song YJ, et al. Mental health status of people isolated due to Middle East Respiratory Syndrome. Epidemiol Health. 2016; 38:e2016048. Published 2016 Nov 5. doi:10.4178/epih.e2016048

5. Chan SM, Chiu FK, Lam CW, Leung PY, Conwell Y. Elderly suicide and the 2003 SARS epidemic in Hong Kong. Int J Geriatr Psychiatry. 2006; 21 (2):113-118. doi:10.1002/gps.1432

6. Williams SN, Armitage CJ, Tampe T, Dienes K. Public perceptions and experiences of social distancing and social isolation during the COVID-19 pandemic: A UK-based focus group study. medRxiv 2020 doi: https://doi.org/10.1101/2020.04.10.20061267 (preprint)

7. https://www.ons.gov.uk/peoplepopulationandcommunity/healthandsocialcare/causesofdeath/bulletins/c oronaviruscovid19relateddeathsbyoccupationenglandandwales/deathsregistereduptoandincluding20apri $\underline{12020}$ [accessed 11/05/20]

8. Zhou F, Yu T, Du R, et al. Clinical course and risk factors for mortality of adult inpatients with COVID-19 in Wuhan, China: a retrospective cohort study; [published correction appears in Lancet. 2020 Mar 28;395(10229):1038]. Lancet. 2020; 395 (10229):1054ロ 1062. doi:10.1016/S01406736(20)30566-3

9. Khunti K, Singh AK, Pareek M, Hanif W. Is ethnicity linked to incidence or outcomes of covid19? BMJ. 2020; 369:m1548. Published 2020 Apr 20. doi:10.1136/bmj.m1548

10. Folkman S. 1997. Positive psychological states and coping with severe stress. Soc. Sci. Med. 45:120721

11. Bults M, Beaujean DJ, de Zwart O, et al. Perceived risk, anxiety, and behavioural responses of the general public during the early phase of the Influenza A (H1N1) pandemic in the Netherlands: results of three consecutive online surveys. BMC Public Health. 2011; 11:2. Published 2011 Jan 3. doi:10.1186/1471-2458-11-2.

12. Salkovskis PM, Rimes KA, Warwick HM, Clark DM. The Health Anxiety Inventory: development and validation of scales for the measurement of health anxiety and hypochondriasis. Psychol Med. 2002;

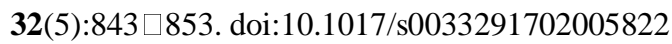


medRxiv preprint doi: https://doi.org/10.1101/2020.05.14.20102012; this version posted May 19, 2020. The copyright holder for this preprint (which was not certified by peer review) is the author/funder, who has granted medRxiv a license to display the preprint in perpetuity.

It is made available under a CC-BY 4.0 International license .

13. Gao J, Zheng P, Jia Y, et al. Mental health problems and social media exposure during COVID-19 outbreak. PLoS One. 2020; 15(4):e0231924. Published 2020 Apr 16.doi:10.1371/journal.pone.0231924

14. Wang J, Mann F, Lloyd-Evans B, Ma R, Johnson S. Associations between loneliness and perceived social support and outcomes of mental health problems: a systematic review. BMC Psychiatry. 2018; 18(1):156. Published 2018 May 29. doi:10.1186/s12888-018-1736-5

15. Leigh-Hunt N, Bagguley D, Bash K, Turner V, Turnbull S, Valtorta N, Caan W. An overview of systematic reviews on the public health consequences of social isolation and loneliness. Public Health. 2017 Nov; 152:157-171. doi: 10.1016/j.puhe.2017.07.035.

16. Pressman SD, Jenkins BN, Moskowitz JT. Positive Affect and Health: What Do We Know and Where Next Should We Go? Ann Rev Psychol. 2019; 70:627—650. doi:10.1146/annurev-psych-010418102955

17. Schotanus-Dijkstra M, ten Have M, Lamers SMA, de Graaf R, Bohlmeijer ET. The longitudinal relationship between flourishing mental health and incident mood, anxiety and substance use disorders. Eur J Public Health. 2017; 27(3):563ॅ568. doi:10.1093/eurpub/ckw202

18. Schotanus-Dijkstra M, Keyes CLM, de Graaf R, ten Have M. Recovery from mood and anxiety disorders: The influence of positive mental health. J Affect Disord. 2019; 252:107 $\square 113$. doi:10.1016/j.jad.2019.04.051

19. Kroenke K, Spitzer RL, Williams JB, Lowe B. The Patient Health Questionnaire Somatic, Anxiety, and Depressive Symptom Scales: a systematic review. Gen Hosp Psychiatry. 2010;32(4):345-59

20. Spitzer RL, Kroenke K, Williams JB, Löwe B. A brief measure for assessing generalized anxiety disorder: the GAD-7. Arch Intern Med. 2006; 166(10):1092-1097. doi:10.1001/archinte.166.10.1092

21. Cohen S, Williamson G. (1988). Perceived Stress in a Probability Sample of the United States. In S. Spacapam \& S. Oskamp (Eds.), The social psychology of health: Claremont Symposium on Applied Social Psychology (pp. 31-67). Newbury Park, CA: Sage.

22. Diener E., Wirtz D, Tov W, et al. (2010). New well-being measures: Short scales to assess flourishing, positive, and negative feelings. Social Indicators Research, 97(2), 143-156. https://doi.org/10.1007/s11205-009-9493-y

23. Kocalevent RD, Hinz A, Brähler E. Standardization of the depression screener patient health questionnaire (PHQ-9) in the general population. Gen Hosp Psychiatry. 2013; 35 (5):551 $\square 555$. doi:10.1016/j.genhosppsych.2013.04.006

24. Warttig SL, Forshaw MJ, South J, White AK. New, normative, English-sample data for the Short Form Perceived Stress Scale (PSS-4). J Health Psychol. 2013; 18(12):1617 $\square 1628$. doi:10.1177/1359105313508346

25. Löwe B, Decker O, Müller S, et al. Validation and standardization of the Generalized Anxiety Disorder Screener (GAD-7) in the general population. Med Care. 2008; 46 (3):266 $\square 274$. doi:10.1097/MLR.0b013e318160d093.

26. https://www.rcpsych.ac.uk/docs/default-source/improving-care/nccmh/iapt/the-iapt-manual--final-republished-7-3-18.pdf?sfvrsn=a192d1af_0 [accessed 5/5/20]

27. https://files.digital.nhs.uk/pdf/q/3/mental health and wellbeing in england full report.pdf [accessed 03/05/20]

28. https://www.marchnetwork.org/research [accessed 04/05/20]

29. Westhoff CL. Breast cancer risk: perception versus reality. Contraception. 1999; 59 (1 Suppl):25S $\square 28$ S. doi:10.1016/s0010-7824(98)00134-6

30. Masi CM, Chen HY, Hawkley LC, Cacioppo JT. A meta-analysis of interventions to reduce loneliness. Per Soc Psychol Rev. 2011; 15 (3):219 $\square 266$. doi:10.1177/1088868310377394

31. O’Connor D, Thayer J, Vedhara K. Stress and Health: A Review of Psychobiological Processes. Annual Review of Psychology (in press).

32. https://thepsychologist.bps.org.uk/we-will-have-live-risk-covid-19-psychology-has-much-say-aboutrisk [accessed 06/05/2020] 